\title{
Modeling and Optimal Control of a Hydrogen Storage System for Wind Farm Output Power Smoothing
}

M. B. Abdelghany*, M. Faisal, D. Liuzza, V. Mariani, L. Glielmo

GRACE, the Group for Research on Automatic Control Engineering Department of Engineering UNIVERSITY OF SANNIO

December 14, 2020
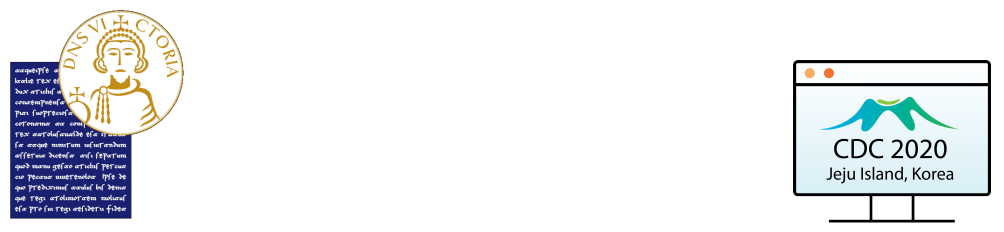
Table of contents

(1) INTRODUCTION

(2) PROBLEM FORMULATION

(3) PROBLEM DESCRIPTION

(4) RESULTS AND DISCUSSIONS 


\section{Paper key contributions}

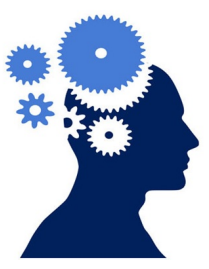

- Operate devices by means of logic commands (ON, OFF,...)

- The modeling recipe is given by MLD + MPC + multiobjective

- Convert/electrify suitable amounts of energy/hydrogen

- The minimum weighted power variation over the prediction horizon is computed first (high priority)

- Then it is used as a constraint in the minimization of the load tracking error stage

- Minimise costs for long term profitability

- Ensure the safety of operations 


\section{Microgrid under investigation}

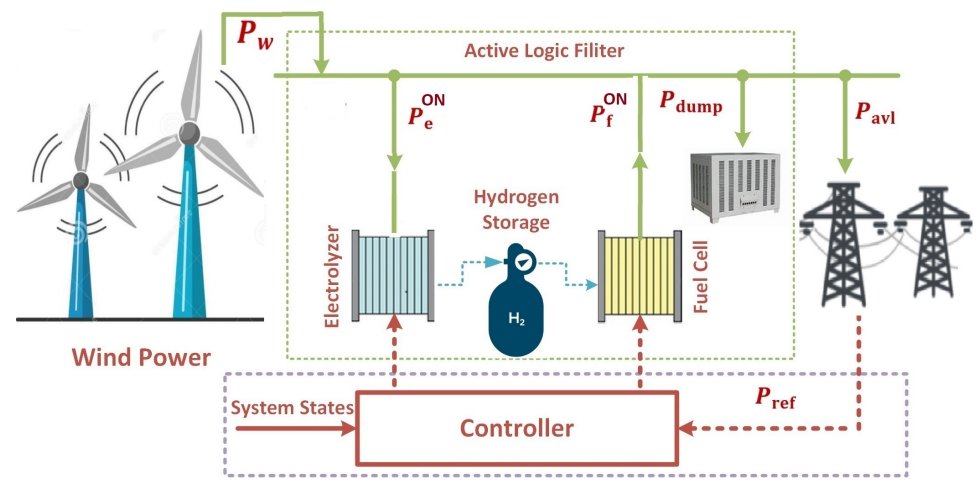




\section{Mixed logical dynamical modeling I}

Automaton for the electrolyzer $(i=e)$ and the fuel cell $(i=f)$.

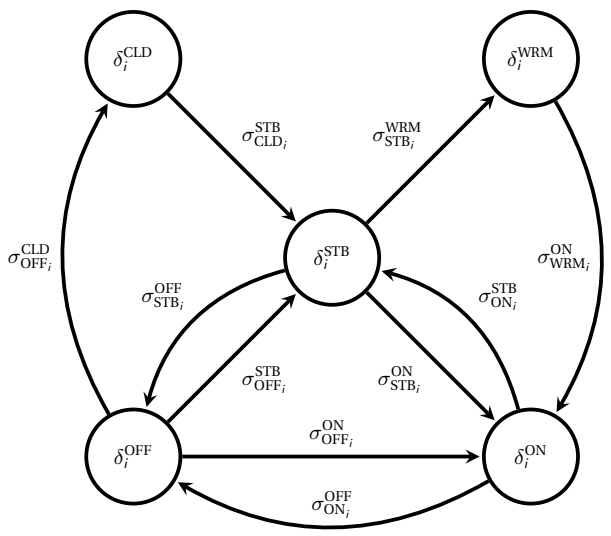

The devices are modeled with 5 state automata described by

- logic states $\delta_{i}^{\alpha}$

- states transitions $\sigma_{\alpha_{i}}^{\beta}$

Logic states and transitions in

- dynamic models

- constraints

- cost functions

- The 5 states of the devices ON, OFF, STB, CLD, WRM characterize the model 


\section{Mixed logical dynamical modeling II}

$P_{i}(k)$ depends on the logic state corresponding to the operating condition of the $i$-th device that is $P_{i}^{\alpha}=P_{i} \delta_{i}^{\alpha}(k)$

$$
\begin{cases}P_{i}(k)=0 & \Longleftrightarrow \delta_{i}^{\mathrm{OFF}}(k)=1 \\ P_{i}(k)=P_{i}^{\mathrm{CLD}} & \Longleftrightarrow \delta_{i}^{\mathrm{CLD}}(k)=1 \\ P_{i}(k)=P_{i}^{\mathrm{STB}} & \Longleftrightarrow \delta_{i}^{\mathrm{STB}}(k)=1 \\ P_{i}(k)=P_{i}^{\mathrm{WRM}} & \Longleftrightarrow \delta_{i}^{\mathrm{WRM}}(k)=1 \\ P_{i}(k) \in\left[P_{i}^{\min }, P_{i}^{\max }\right] & \Longleftrightarrow \delta_{i}^{\mathrm{ON}}(k)=1\end{cases}
$$

Two (boolean) slack variables $z_{i}^{\geq \gamma}(k), z_{i}^{\leq \bar{\gamma}}(k)$ are introduced

$$
\begin{aligned}
& z_{i}^{\geq \gamma}(k)= \begin{cases}1 & P_{i}(k) \geq \gamma \\
0 & P_{i}(k)<\gamma\end{cases} \\
& z_{i}^{\leq \bar{\gamma}}(k)= \begin{cases}0 & P_{i}(k)>\bar{\gamma} \\
1 & P_{i}(k) \leq \bar{\gamma}\end{cases}
\end{aligned}
$$

$(\gamma, \bar{\gamma}) \in\left\{(0,0),\left(P_{i}^{\mathrm{CLD}}, P_{i}^{\mathrm{CLD}}\right),\left(P_{i}^{\mathrm{STB}}, P_{i}^{\mathrm{STB}}\right),\left(P_{i}^{\mathrm{WRM}}, P_{i}^{\mathrm{WRM}}\right),\left(P_{i}^{\min }, P_{i}^{\max }\right)\right\}$ 


\section{Mixed logical dynamical modeling III}

- MLD constraints of the devices logic states

$P_{i}(k)$ can be linked to $\delta_{i}^{\alpha}$ with inequalities through $z_{i}^{\geq \gamma}, z_{i}^{\leq \bar{\gamma}}$ (Big M-reformulation)

$$
\begin{array}{rcrl}
P_{i}(k)-\gamma<M z_{i}^{\geq \gamma}(k) & -P_{i}(k)+\bar{\gamma}<M z_{i}^{\leq \bar{\gamma}}(k) \\
-P_{i}(k)+\gamma \leq M\left(1-z_{i}^{\geq \gamma}(k)\right) & P_{i}(k)-\bar{\gamma} \leq M\left(1-z_{i}^{\leq \bar{\gamma}}(k)\right) \\
\left(1-\delta_{i}^{\alpha}(k)\right)+z_{i}^{\geq \gamma}(k) \geq 1 & \left(1-\delta_{i}^{\alpha}(k)\right)+z_{i}^{\leq \bar{\gamma}}(k) \geq 1
\end{array}
$$

The following constrain must be added

$$
\sum_{\alpha} \delta_{i}^{\alpha}(k)=1
$$

where $\alpha \in \mathcal{A}, \mathcal{A}=\{\mathrm{OFF}, \mathrm{CLD}, \mathrm{STB}, \mathrm{WRM}, \mathrm{ON}\}$ 


\section{Mixed logical dynamical modeling IV}

- MLD constraints of the devices state transitions

The AND operator $(\wedge)$ can be written in terms of inequalities

$$
\mu_{3}=\mu_{1} \mu_{2} \Longleftrightarrow\left\{\begin{array}{l}
\mu_{3} \leq \mu_{1} \\
\mu_{3} \leq \mu_{2} \\
\mu_{3} \geq \mu_{1}+\mu_{2}-1
\end{array}\right.
$$

where $\mu_{j} \in\{0,1\}, \quad j \in\{1,2,3\}, \mu_{1}=\delta_{i}^{\alpha}(k-1), \mu_{2}=\delta_{i}^{\beta}(k)$ and $\mu_{3}=\sigma_{\alpha, i}^{\beta}(k)$. All the inadmissible transitions, i.e., those not shown in automata, are set to 0

$$
\begin{aligned}
& \sigma_{\mathrm{OFF}, i}^{\mathrm{STB}}(k)=\sigma_{\mathrm{OFF}, i}^{\mathrm{WRM}}(k)=\sigma_{\mathrm{OFF}, i}^{\mathrm{ON}}(k)=\sigma_{\mathrm{CLD}, i}^{\mathrm{OFF}}(k) \\
= & \sigma_{\mathrm{CLD}, i}^{\mathrm{WRM}}(k)=\sigma_{\mathrm{CLD}, i}^{\mathrm{ON}}(k)=\sigma_{\mathrm{STB}, i}^{\mathrm{ON}}(k)=\sigma_{\mathrm{STB}, i}^{\mathrm{CLD}}(k) \\
= & \sigma_{\mathrm{WRM}, i}^{\mathrm{OFF}}(k)=\sigma_{\mathrm{WRM}, i}^{\mathrm{CL}}(k)=\sigma_{\mathrm{WRM}, i}^{\mathrm{STB}}(k)=\sigma_{\mathrm{ON}, i}^{\mathrm{CLD}}(k) \\
= & \sigma_{\mathrm{ON}, i}^{\mathrm{WRM}}(k)=0
\end{aligned}
$$




\section{Mixed logical dynamical modeling $\mathrm{V}$}

- Operating constraints

$$
\begin{aligned}
& \delta_{i}^{\mathrm{CLD}}(k)-\delta_{i}^{\mathrm{CLD}}(k-1) \leq \delta_{i}^{\mathrm{CLD}}\left(\tau^{\mathrm{CLD}}\right) \\
& \delta_{i}^{\mathrm{CLD}}(k)+\cdots+\delta_{i}^{\mathrm{CLD}}\left(k-T^{\mathrm{CLD}}\right) \leq T^{\mathrm{CLD}} \\
& \tau^{\mathrm{CLD}}=k+1, \ldots, k+T^{\mathrm{CLD}} \\
& \delta_{i}^{\mathrm{WRM}}(k)-\delta_{i}^{\mathrm{WRM}}(k-1) \leq \delta_{i}^{\mathrm{WRM}}\left(\tau^{\mathrm{WRM}}\right) \\
& \delta_{i}^{\mathrm{WRM}}(k)+\cdots+\delta_{i}^{\mathrm{WRM}}\left(k-T^{\mathrm{WRM}}\right) \leq T^{\mathrm{WRM}} \\
& \tau^{\mathrm{WRM}}=k+1, \ldots, k+T^{\mathrm{WRM}}
\end{aligned}
$$

- Ramp up constraints

$$
\left|\left(P_{i}(k+1)-P_{i}(k)\right) \delta_{i}^{\mathrm{ON}}\right| \leq R_{i}
$$




\section{Mixed logical dynamical modeling $\mathrm{VI}$}

- Hydrogen dynamics

$$
H(k+1)=H(k)+\eta_{e}(k) P_{e}(k) \delta_{e}^{\mathrm{ON}}(k) T_{s}-\frac{P_{f}(k) \delta_{f}^{\mathrm{ON}}(k) T_{s}}{\eta_{f}(k)}
$$

- Operating ranges

$$
\begin{aligned}
& P_{i}^{\min } \leq P_{i}(k) \leq P_{i}^{\max } \\
& H^{\text {min }} \leq H(k) \leq H^{\max }
\end{aligned}
$$

- Power balance

$$
P_{w}(k)-P_{e}(k) \delta_{e}^{\mathrm{ON}}(k)+P_{f}(k) \delta_{f}^{\mathrm{ON}}(k)-P_{\mathrm{avl}}(k)-P_{\mathrm{dump}}(k)=0
$$




\section{ESS operating costs}

$$
\begin{aligned}
J_{i}(k+j):= & \left(\frac{S_{\mathrm{rep}, i}}{\mathrm{NH}_{i}}+\mathrm{C}_{i}^{\mathrm{OM}}\right) \delta_{i}^{\mathrm{ON}}(k+j) \\
& +\mathrm{C}_{\mathrm{ON}, i}^{\mathrm{OFF}} \sigma_{\mathrm{ON}, i}^{\mathrm{OFF}}(k+j) \\
& +\mathrm{C}_{\mathrm{CLD}, i}^{\mathrm{STB}} \sigma_{\mathrm{CLD}, i}^{\mathrm{STB}}(k+j) \\
& +\mathrm{C}_{\mathrm{STB}, i}^{\mathrm{OFF}} \sigma_{\mathrm{STB}, i}^{\mathrm{OFF}}(k+j) \\
& +s(k+j) P_{i}^{\mathrm{STB}} \delta_{i}^{\mathrm{STB}}(k+j) \\
& +s(k+j) P_{i}^{\mathrm{CLD}} \delta_{i}^{\mathrm{CLD}}(k+j) \\
& +s(k+j) P_{i}^{\mathrm{WRM}} \delta_{i}^{\mathrm{WRM}}(k+j)
\end{aligned}
$$

where

- $S_{\text {rep }, i}$ : the $i$-device stack replacement cost

- $\mathrm{C}_{i}^{\mathrm{OM}}$ : the $i$-device O\&M cost

- $\mathrm{C}_{\mathrm{ON}_{i}}^{\mathrm{OFF}}, \mathrm{C}_{\mathrm{CLD}, i}^{\mathrm{STB}}, \mathrm{C}_{\mathrm{STB}_{i}}^{\mathrm{OFF}}$ : the $i$-th device cycle costs

- $P_{i}^{S T B}(k)$ : the power at standby

- $s(k)$ : the power spot price 


\section{Cost functions}

- Power smoothing cost function

$$
J_{s}(k):=\sum_{j=0}^{T-1} \sum_{\tau=1}^{\tau_{B}} \omega^{k+j, \tau} y^{k+j, \tau},
$$

where $y^{k+j, \tau}$ is the power increment such that

- $y^{k+j, \tau} \geq 0$

- $y^{k+j, \tau} \geq\left|P_{\text {avl }}(k+j)-P_{\text {avl }}(k+j-\tau)\right|-\bar{y}^{\tau}$

with $\bar{y}^{\tau}$ is a given power reference depending on $\tau$

- Load tracking cost function

$$
J_{l}(k):=\frac{1}{T} \sum_{j=0}^{T-1}\left(P_{\text {avl }}(k+j)-P_{\text {ref }}(k+j)\right)^{2} .
$$

- Global cost function

$$
J(k):=\sum_{j=0}^{T-1} \rho_{l} J_{l}(k+j)+\rho_{e} J_{e}(k+j)+\rho_{f} J_{f}(k+j),
$$




\section{Multi-objective optimization I}

$$
\min _{\mathcal{C}_{k}}\left\{J_{s}(k), J(k)\right\}
$$

Subject to

Discrete logical states constraints,

Mode transitions constraints,

Physical constraints,

Hydrogen level dynamics,

Power smoothing constraints.

$\min _{\mathcal{C}_{k}} J_{s}(k)$

s.t.

All constraints.

$$
\min _{\mathcal{C}_{k}} J(k)
$$

s.t.

All constraints,

$$
J_{s}(k) \leq J_{s}^{*} \text {. }
$$




\section{Multi-objective optimization II}

- At each time step $k$, given the initial state $H(k)$, the MPC provides the optimal control sequences

$$
\mathcal{C}_{k}:=\left\{\boldsymbol{P}_{\mathrm{i}, k}^{T-1}, \boldsymbol{P}_{\mathrm{avl}, k}^{T-1}, \mathrm{P}_{\mathrm{dump}, k}^{T-1}, \boldsymbol{\delta}_{\mathrm{i}, k}^{\alpha, T-1}, \boldsymbol{\sigma}_{\alpha, \mathrm{i}, k}^{\beta, T-1}, \boldsymbol{z}_{\mathrm{i}, k}^{\gamma, T-1}\right\},
$$

where

$$
\begin{aligned}
\boldsymbol{P}_{i, k}^{T-1} & =\left(P_{i}(k), \ldots, P_{i}(k+T-1)\right)^{\top} \\
\boldsymbol{P}_{\mathrm{avl}, k}^{T-1} & =\left(P_{\mathrm{avl}}(k), \ldots, P_{\mathrm{avl}}(k+T-1)\right)^{\top} \\
\boldsymbol{P}_{\mathrm{dump}, k}^{T-1} & =\left(P_{\mathrm{dump}}(k), \ldots, P_{\mathrm{dump}}(k+T-1)\right)^{\top} \\
\boldsymbol{\delta}_{i, k}^{\alpha, T-1} & =\left(\delta_{i}^{\alpha}(k), \ldots, \delta_{i}^{\alpha}(k+T-1)\right)^{\top} \\
\boldsymbol{\sigma}_{\alpha_{i, k}}^{\beta, T-1} & =\left(\sigma_{\alpha_{i}}^{\beta}(k), \ldots, \sigma_{\alpha_{i}}^{\beta}(k+T-1)\right)^{\top} \\
\boldsymbol{z}_{i, k}^{\gamma, T-1} & =\left(z_{i}^{\gamma}(k), \ldots, z_{i}^{\gamma}(k+T-1)\right)^{\top}
\end{aligned}
$$




\section{Results I}

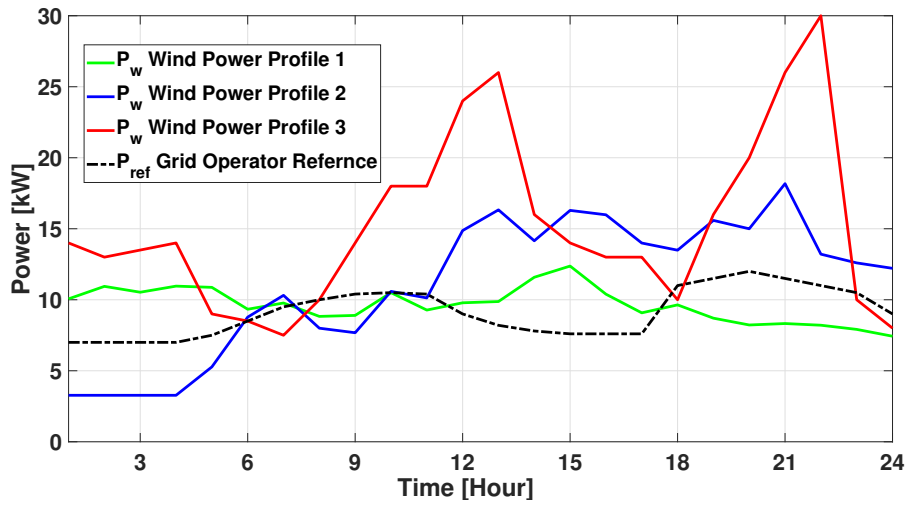

Figure: Wind and operator power profiles 


\section{Results II}

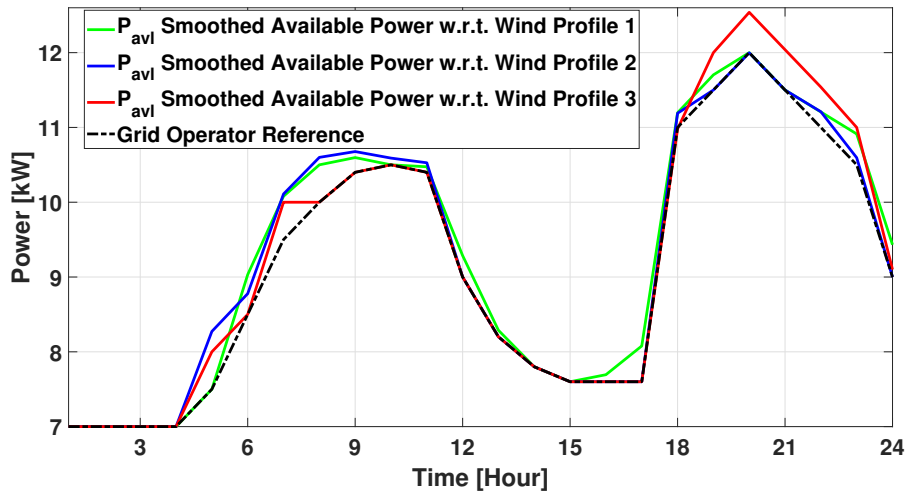

Figure: Smoothed available power profiles 


\section{Results III}

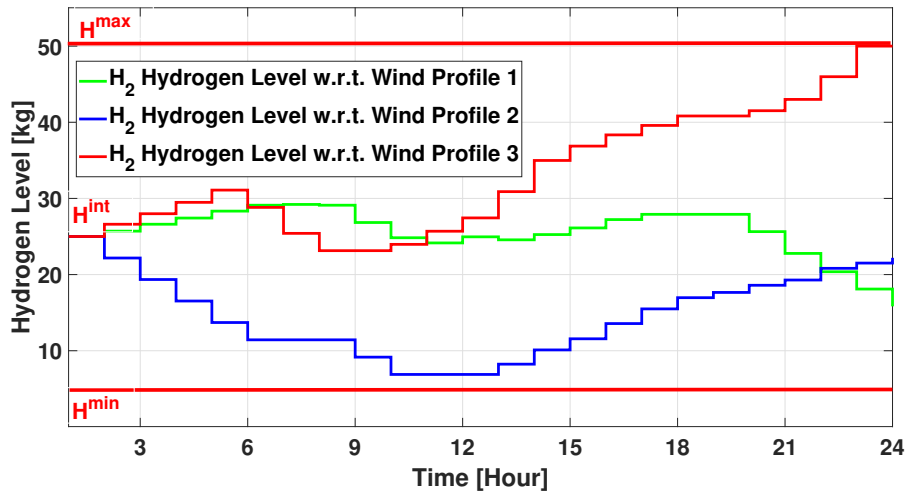

Figure: Hydrogen levels 


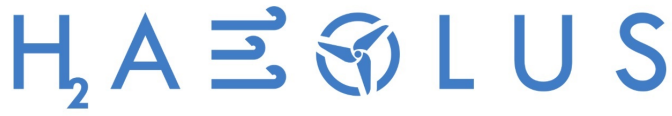

\section{Hydrogen-Aeolic Energy with Optimised eLectrolysers Upstream of Substation}

This project has received funding from the Fuel Cells and Hydrogen 2 Joint Undertaking under the European Union's Horizon 2020 research and innovation programme under grant agreement № 779469.
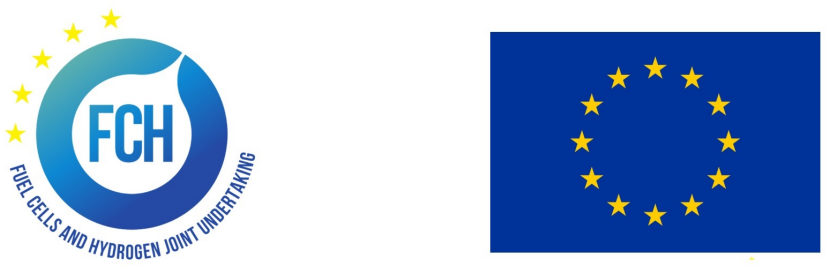
Thank you for your attention 